\title{
Incidental retroperitoneal paraganglioma in patient candidate to radical prostatectomy: Concurrent surgical treatments by robotic approach
}

\author{
Matteo Ferrari, MD; Mattia Sangalli, MD; Matteo Zanoni, MD; Massimo Ghezzi, MD; Fabio Fabbri, MD; \\ Francesco Sozzi, MD; Patrizio Rigatti, MD; Andrea Cestari, MD
}

Department of Urology, Advanced Urotechnology Center, Istituto Auxologico Italiano, Milan, Italy

Cite as: Can Urol Assoc J 2015;9(7-8):E539-41. http://dx.doi.org/10.5489/cuaj.2501

Published online July 17, 2015.

\section{Abstract}

We report a case of a 75-year-old male with biopsy-proven prostate cancer and candidate for radical prostatectomy. The patient's medical history includes hypertension and atrial fibrillation in prophylactic treatment; however, he was suffering from recurrent paroxysmal episodes of supraventricular tachycardia. Abdominal magnetic resonance performed for prostate cancer staging detected a non-lymphatic inter-cavo-aortic mass of $42 \times 37 \times 43 \mathrm{~cm}$. Results of biochemical screening confirmed the clinical diagnosis of symptomatic paraganglioma. The patient was subjected in a single robotic session for concurrent excision of the inter-aortocaval mass and radical prostatectomy with bilateral pelvic lymphnode dissection. During the procedure, there were no anesthesiological or surgical complications. The postoperative course was uneventful and the patient was discharged on postoperative day 5. Six months after surgery, his prostate-specific antigen level was undetectable and the abdominal magnetic resonance imaging was negative for local recurrence or metastasis of paraganglioma. No more episodes of tachycardia were reported or antihypertensive therapy was necessary.

\section{Introduction}

Paraganglioma is a rare neuroendocrine tumour secreting catecholamines. This characteristic, together with its extraadrenal localization at any level of sympathetic and parasympathetic tissue, makes its treatment a challenge from the surgical and anesthesiological standpoint. Surgical treatment of paraganglioma may become even more difficult in rare clinical situations in the presence of synchronous tumours.

\section{Case report}

We present the case of a 75-year-old man with a biopsyproven diagnosis of bilateral prostate cancer and candidate to treatment with curative intent. The patient's history was notable for hypertension treated with amlodipine and atrial fibrillation treated with pharmacologic cardioversion many years before, in prophylactic treatment with flecainide acetate. However, the patient was suffering from recurrent paroxysmal episodes of supraventricular tachycardia.

Abdominal staging with magnetic resonance imaging (MRI) detected an inter-cavo-aortic mass of $42 \times 37 \times 43 \mathrm{~cm}$ between the inferior mesenteric artery and the iliac bifurcation (Fig. 1). No abdominal lymphadenopathy or parenchymal lesions were found, as bone scintigraphy was negative for metastasis. Biochemical screening for neuroendocrine diseases showed an increased plasma level of norepinephrine $(514 \mathrm{pg} / \mathrm{mL})$ and chromogranin $\mathrm{A}(114.3 \mathrm{ng} / \mathrm{mL})$. The clinical diagnosis led us to suspect symptomatic paraganglioma associated with localized prostate cancer: both with an indication to surgical treatment. Fundoscopy was negative for hypertensive retinopathy.

To control possible cardiovascular crisis related to catecholamine release, 7 days before surgery patient started treatment with phenoxybenzamine, metoprolol, and hydration therapy to restore intravascular volume. The patient was subjected in a single robotic session for concurrent excision of the inter-aorto-caval mass and radical prostatectomy with bilateral pelvic lymph-node dissection (PLND). On the operating table, the patient was placed in the supine Trendelemburg position and surgical ports were positioned according to our consolidated scheme. ${ }^{1}$

We started with resecting the paraganglioma. After opening the posterior parietal peritoneum, we then isolated the 


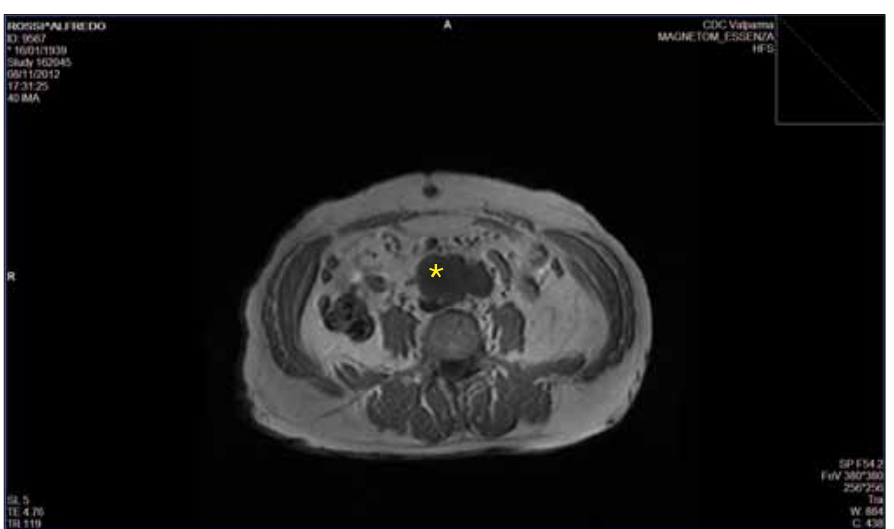

Fig. 1. Axial T1 magnetic resonance image showing tumoral mass at the level of iliac bifurcation (asterisk).

mass avoiding any potential traumatic maneuver and controlled the vascular supply using Hem-o-lok clips (Fig. 2). The mass was completely resected and then placed in an endobag. Subsequently we proceeded to perform the radical prostatectomy and bilateral PLND. The total operative time was 240 minutes, of which 60 minutes was required for the paraganglioma resection. Blood loss was $150 \mathrm{~mL}$. There were no anesthesiological or surgical complications. The postoperative course was uneventful with normalization of blood pressure without the need for medication. The patient resumed a normal diet on postoperative day 1 , and was discharged on postoperative day 5 .

The definitive histologic diagnosis was extra-adrenal paraganglioma. Prostate cancer pathology revealed an intraprostatic bilateral carcinoma Gleason score $3+3$ with lymph nodes removed negative for metastasis. Six months after surgery, his prostate-specific antigen was undetectable and his abdominal MRI was negative for local recurrence or metastasis. The patient had no more episodes of tachycardia and antihypertensive therapy was unnecessary.

\section{Discussion}

Paraganglioma is a rare chromaffin cell tumour from extraadrenal symphatetic tissue and is mostly located in the abdomen. Intrabdominal sympatethic paraganglioma is usually endocrinologically active, secreting cathecolamines responsible for a variety of non-specific cardiovascular and autonomic signs and symptoms.

Our patient had an incidental diagnosis of paraganglioma, performed during routine abdominal staging while he was a candidate for RP. His history includes two of the most frequent signs of pheochromocytoma/paraganglioma, hypertension, and tachycardia. Increased levels of plasma chromogranin A and norepinephrine confirmed our clinical suspicion.

Treatment for paraganglioma is similar to that for adrenal counterpart pheochromocytoma; complete surgical excision is needed to prevent life-threatening cardiovascular crisis or malignant behaviour. Paraganglioma was associated with an increased risk of developing metastatic disease compared with adrenal pheochromocytoma. ${ }^{2}$ The tumour dimension was $>5 \mathrm{~cm}$, high 3-methoxytyramina plasma level, and SDHB gene mutation were predictive factors. ${ }^{3}$ Our patient's disease did not show these clinical features.

The mini-invasive laparoscopic approach is currently the reference technique to perform a total or partial adrenalectomy in patients affected by pheochromocytoma. Recent data have shown that robotic resection is a non-inferior technique regarding safety and efficacy. ${ }^{4}$ Similarly, concerning paraganglioma surgery, the mini-invasive laparoscopic approach is the current treatment of choice. Goers and colleagues ${ }^{5}$ reported a safety profile with low morbidity and outcome similar to adrenal pheochromocytomas; conversely in this series performed through lateral transperitoneal approach, the surgical time was significantly longer.

In this context, tumour size, localization, invasiveness, the presence of multiple or metastatic mass were factors lim-
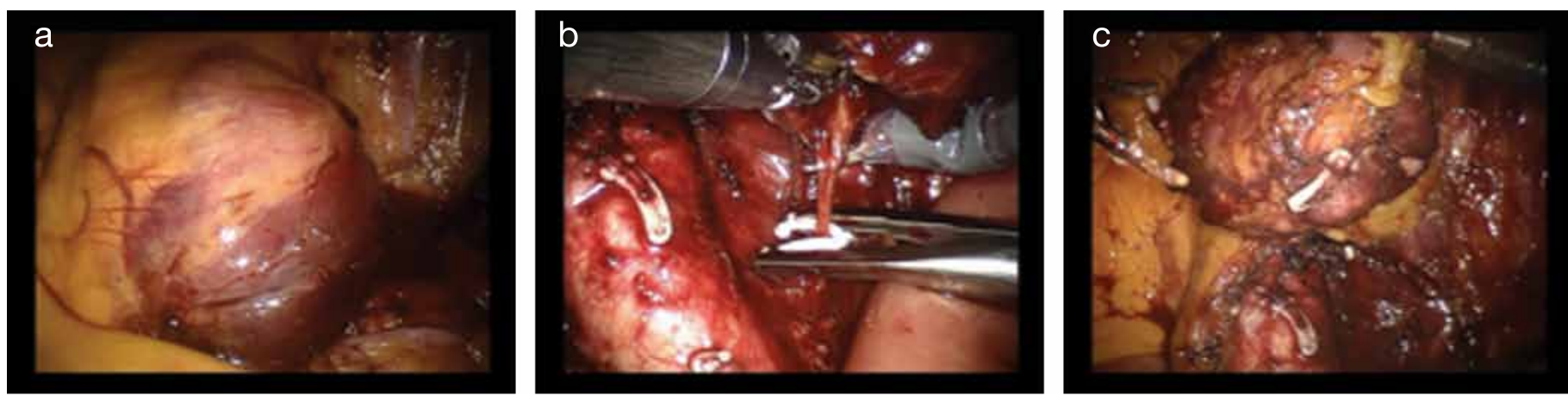

Fig. 2. Intraoperative images showing (a) the mass grew in the retroperitoneum; (b) fine dissection of the vascular tumoral pedicles using Hem-0-lok clips; and (c) the paraganglioma completely resected. 
iting the laparoscopic approach in favour of open surgery. Unlike the pheochromocytoma, data on surgery for paraganglioma with a robotic approach are limited and related only to few case reports. ${ }^{6-9}$

The largest paraganglioma resected by robotic technique was reported by Draaisma and colleagues; ${ }^{6}$ a $7 \times 5.1 \times 3.5$ $\mathrm{cm}$ mass in the left para-aortic region was resected by transperitoneal approach with the patient in a lateral decubitus position. Conversely, Cochetti and colleagues ${ }^{8}$ first reported the use of retroperitoneal approach for robotic resection of a $3 \times 3-\mathrm{cm}$ mass between the left renal vein and the aorta.

Our case report is unique and relevant because the paraganglioma resection was carried out simultaneously with radical prostatectomy and PLND, with considerable advantage for the patient. Our coded standard surgical ports disposition for robotic $\mathrm{RP}^{1}$ has been effective in allowing the surgeon to act on more cranial surgical beds without difficulty. Moreover, resection performed by a high-experience robotic surgeon was carried out in a very limited surgical time. Robotic surgery has clear advantages: gentle and precise dissection of paraganglioma, often difficult to reach for anatomical position, and lower traumatic impact on tumoral mass, preventing potentially dangerous intraoperative catecholamine secretion.

\section{Conclusion}

We present, what we consider the first reported case, of combined surgical treatment of prostate cancer and retroperitoneal paraganglioma performed with a robotic technique. This single-session double surgery, even more critical with regard to the surgical technique and anesthesiological management of paraganglioma, was feasible and free of perioperative complications if performed by a highly experienced surgeon in a robotic centre. In this context, robotic surgery provides significant advantages in the surgical treatment of unusual clinical cases. With more series, we believe the robotic approach can become reference technique to treat this rare disease.

Competing Interests: The authors declare no competing financial or personal interests.

This paper has been peer-reviewed.

\section{References}

1. Cestari A, Buffi NM, Scapaticci E, et al. Simplifying patient positoning and port placement during robotic-assisted laparoscopic prostatectomy. Eur Urol 2010;57:530-3. http://dx.doi.org/10.1016/i. eururo.2009.11.028

2. Martucci V, Pacak K. Pheochromocytoma and paraganglioma: Diagnosis, genetics, management and treatment. Curr Probl Cancer 2014;38:7-41. http://dx.doi.org/10.1016/i.currproblcancer.2014.01.001

3. Van Berkel A, Lenders JWM, Timmers HJLM. Biochemical diagnosis of phaeochromocytoma and paraganglioma. Eur J Endocrinol 2014;170:109-19. http://dx.doi.org/10.1530/EJE-13-0882

4. Aliyev $S$, Karabulut K, Agcaoglu 0 , et al. Robotic versus laparoscopic adrenalectomy for pheochromocytoma. Ann Surg Oncol 2013;20:4190-4. http://dx.doi.org/10.1245/s10434-013-3134-z

5. Goers TA, Abdo M, Moley JF, et al. Outcomes of resection of extra-adrenal pheochromocytomas/ paragangliomas in the laparoscopic era: A comparison with adrenal pheochromocytoma. Surg Endosc 2013;27:428-33. http://dx.doi.org/10.1007/s00464-012-2451-9

6. Draaisma WA, Van Hillegersberg R, Borel Rinkes IH, et al. Robot-assisted laparoscopic resection of a large paraganglioma: A case report. Surg Laparosc Endosc Percutan Tech 2006;16:362-5. http://dx.doi. org/10.1097/01.sle.0000213717.19344.1b

7. Lehrfeld T, Natale R, Sharma S, et al. Robot-assisted excision of a retroperitoneal mass between the left renal artery and vein. JSLS 2010;14:447-9. http://dx.doi.org/10.4293/108680810X12924466006927

8. Cochetti $G$, Barillaro $F$, Boni $A$, et al. Robot assisted laparoscopic excision of a paraganglioma: New therapeutic approach Int Braz J Urol 2014;40:279-80. http://dx.doi.org/10.1590/S1677-5538. IBJU.2014.02.21

9. Park JS, Lee KY, Kim JK, et al. The first laparoscopic resection of extra-adrenal pheohromocytoma using the aVinci robotic system. J Laparoendosc Adv Surg Tech A 2009;19:63-5. http://dx.doi.org/10.1089/ lap.2008.0019

Correspondence: Dr. Matteo Ferrari, Advanced Urotechnology Center, Istituto Auxologico Italiano, via Mercalli 30, 20122 Milan, Italy; em.ferrari@alice.it 